\title{
Análise retrospectiva de denúncias de maus-tratos contra animais na região de Curitiba, Estado do Paraná, utilizando critérios de bem-estar animal
}

\author{
Retrospective analysis of animal abuse in the region of Curitiba, State of Paraná, Southern \\ Brazil, using animal welfare criteria
}

\author{
Janaina HAMMERSCHMIDT' ${ }^{1}$;Carla Forte Maiolino MOLENTO ${ }^{1}$ \\ ${ }^{1}$ Laboratório de Bem-estar Animal (LABEA/UFPR), Curitiba-PR, Brasil
}

\begin{abstract}
Resumo
A relação entre seres humanos e animais deve ser positiva. No entanto, interações negativas também têm sido descritas, como os relatos de ocorrências de maus-tratos contra animais. Os objetivos deste trabalho foram estudar o perfil de denúncias de maus-tratos contra animais registradas nas Sociedades Protetoras dos Animais de Campo Largo e de Curitiba e na Rede de Defesa e Proteção Animal de Curitiba, e analisar a pertinência da ação de profissionais capacitados em tais processos. Os elementos apurados foram espécies animais envolvidas e descrição das denúncias a fim de coletar as informações pertinentes e classificá-las de acordo com as causas e com as Cinco Liberdades. Das denúncias, $82,9 \%$ envolveram cães. As causas mais comuns foram abandono (16,2\%), não fornecimento de alimento e água $(15,7 \%)$, morte $(14,7 \%)$ e ausência de atendimento veterinário (13,5\%). A maioria das ocorrências fez referência às liberdades sanitária $(71,8 \%)$ e nutricional $(28,2 \%)$, embora a avaliação técnica tenha revelado o comprometimento comum da liberdade psicológica $(93,5 \%)$. Os resultados sugerem que a colaboração de profissional capacitado em bemestar animal pode subsidiar o aprimoramento das deliberações em casos de suspeita de maus-tratos aos animais.
\end{abstract}

Palavras-chave: Cães. Cinco liberdades. Gênero do denunciado. Proteção animal. Sofrimento.

\begin{abstract}
The human-animal relationship should be positive; however, negative interactions are frequently registered, some of which can be found as reports of incidents of animal abuse. The aim of this study was to survey the history of complaints of animal abuse recorded in the Animal Protection Society of Campo Largo and Curitiba and in the Animal Defense and Protection Network of Curitiba, Paraná, and to analyze the relevance of veterinary action in such cases. The data collected were animal species involved, description of complaints and relevant information to classify them according to the causes and the Five Freedoms. Of the allegations, $82.9 \%$ involved dogs. The most common causes were abandonment (16.2\%), failure to provide food and water (15.7\%), death (14.7\%) and lack of veterinary care (13.5\%). Most of the cases referred to the freedom from pain, injury and disease, and the freedom from hunger and thirst, although the technical evaluation has revealed a frequent impact on psychological freedom. The results suggest that the collaboration of a professional specialized in animal welfare can enhance the deliberations in cases of suspected animal abuse.
\end{abstract}

Keywords: Abuser gender. Animal protection. Dogs.Five freedoms. Suffering.

\section{Introdução}

Embora a relação entre seres humanos e animais deva ser positiva para ambos, interações negativas também são observadas, como os relatos de atos de maus-tratos em animais. Tais atos devem ser investigados e julgados,porém, o sucesso destas iniciativas depende, entre outros, de relatos ou denúncias de atos violentos ou cruéis ${ }^{1}$. Para que ocorra a denúncia, é necessário que a sociedade esteja sensível às práticas de maus-tratos contra animais e tenha acesso aos locais para acolhimento de denúncias. No Brasil, a implantação de delegacias especializadas em proteção animal

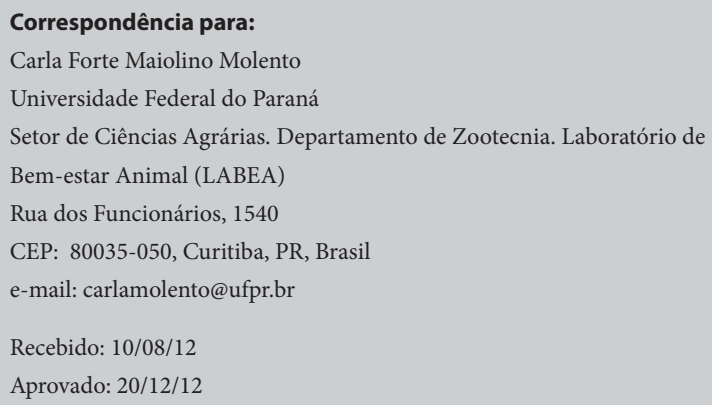


está se intensificando, com destaque para Campinas, em São Paulo, pelo seu pioneirismo ${ }^{2}$. Além do relato, para execução das sanções pertinentes, é necessário um sistema legislativo mais detalhado sobre o assunto. No Brasil, a Lei de Crimes Ambientais 9.605 de 1998 é o principal instrumento jurídico em defesa dos animais ${ }^{3}$. Entretanto, ao ser considerado um crime de pequena punibilidade ${ }^{4}$, as investigações podem não ser pormenorizadas tecnicamente e informações podem ser limitadas.

Deste modo, existem oportunidades concretas de melhoria em termos de compreensão da situação de maus-tratos aos animais no Brasil. O objetivo deste trabalho foi compreender o perfil de denúncias de maus-tratos em animais registradas em três instituições envolvidas com proteção animal nos municípios de Campo Largo e Curitiba, ambas no estado do Paraná, para entender as características de denunciantes, denunciados, espécies envolvidas e causas mais comuns de denúncias, assim como avançar no conhecimento quanto à pertinência da colaboração de profissionais capacitados em bem-estar animal em tais processos.

\section{Material e Métodos}

O estudo retrospectivo foi realizado nos municípios de Campo Largo e Curitiba, estado do Paraná. As informações foram provenientes da Sociedade Protetora dos Animais de Campo Largo (SPACL), no período de 2001 a 2009, da Sociedade Protetora dos Animais de Curitiba (SPAC), no período de 2003 a 2011, e da Rede de Defesa e Proteção Animal de Curitiba (RDPAC), no período de julho de 2009 a agosto de 2010. A SPACL e a SPAC atuam em verificação, registro e acompanhamento de denúncias junto aos órgãos competentes, promovendo o encaminhamento de ocorrências para processo judicial somente quando as consideram pertinentes, com base em julgamento pessoal. Os casos estudados foram aqueles de denúncias registradas em órgãos oficiais por meio de Boletim de Ocorrência (BO), uma vez que os demais atendimentos não foram registrados. $\mathrm{A} \mathrm{RDPAC}^{5}$ tem por objetivo atuar na proteção animal, recebendo denúncias por meio do serviço Fale Conosco. Além da RDPAC, existem no mínimo cinco outros órgãos para acolhimento de denúncias: a Delegacia de Proteção ao Meio Ambiente, o Ministério Público, a Força Verde, o Instituto Brasileiro do Meio Ambiente e dos Recursos Naturais Renováveis (IBAMA) e o Instituto Ambiental do Paraná (IAP). Portanto, as denúncias estudadas representam uma visão parcial das denúncias na cidade de Curitiba, cuja dimensão completa não foi possível estimar pela dificuldade de acesso aos dados de outras entidades. As informações obtidas foram espécie e número de animais envolvidos, local de ocorrência, sentença determinada nos casos de fechamento do processo judicial e descrição breve da denúncia. A localização geográfica das denúncias foi realizada a partir do endereço constante nos registros.

Foram interpretados os relatos dos denunciantes e todos os documentos compilados no processo, como boletins de ocorrência das delegacias locais, cópias de depoimentos de testemunhas, cópias do inquérito policial e ação penal e pareceres de médicos veterinários. Para melhor entendimento dos motivos e gravidade das ocorrências, estas foram classificadas em onze categorias de acordo com suas causas, sendo que uma mesma denúncia por vezes se enquadrou em mais de uma categoria. As onze categorias foram abandono; não fornecimento de alimento e água; morte do animal; ausência de atendimento veterinário; trauma físico, agressão; animal com restrição de espaço; outros fatores de inadequação ambiental; cães de guarda de empresas, negligência; animal com acesso à rua sozinho; abuso, uso excessivo e outros. As denúncias também foram classificadas segundo as Cinco Liberdades do bem-estar animal ${ }^{6}$, adaptadas por Molento $^{7}$ em liberdades nutricional, ambiental, sanitária, comportamental e psicológica. As Cinco Liberdades constituem uma abordagem lógica, ampla e estrutura- 
da para diagnóstico de bem-estar animal em campo. O objetivo foi identificar o grau de restrição de cada Liberdade com base no relato do denunciante e na interpretação técnica das ocorrências. Em uma mesma denúncia poderia haver o comprometimento de mais de uma liberdade.

Buscou-se interpretar qual o encaminhamento mais adequado de acordo com as classificações estudadas, no sentido de entender se o diagnóstico técnico de bem-estar animal pode aprimorar a finalização jurídica dos processos legais. Os dados foram estudados por meio de estatística descritiva.

\section{Resultados e Discussão}

Na SPACL, na SPAC e na RDPAC foram registradas 64, 72 e 104 denúncias de maus-tratos, com média anual de 7; 8 e 104 ocorrências, respectivamente. Tal diferença talvez esteja relacionada ao fato de que nas primeiras instituições somente os casos considerados de maior gravidade foram registrados, assim como à facilidade para realização da denúncia na RDPAC via correio eletrônico. A SPACL e a SPAC recebem um número muito maior de denúncias, porém não há registro e há falta de pessoal para auxiliar no atendimento das ocorrências e encaminhar os casos pertinentes à justiça. Na RDPAC as denúncias não foram encaminhadas para órgão oficial, uma vez que tal atividade ainda não havia sido iniciada nessa entidade.

A figura 1 ilustra a distribuição geográfica dos casos em Campo Largo (A) e em Curitiba (B). Em Campo Largo, as denúncias concentraram-se em torno do centro da cidade. A região rural, com menor número de moradores, não apresentou focos de denúncias, talvez por falta de relato ou menor prática de maus-tratos contra animais por pessoas do ambiente rural em comparação com as do ambiente urbano ${ }^{8,9}$. Ressalta-se a grande concentração de denúncias em locais mais próximos à sede da SPACL, talvez pelo maior esclarecimento nessa região sobre os trabalhos desenvolvidos pela entidade. Além disso, no centro da cidade

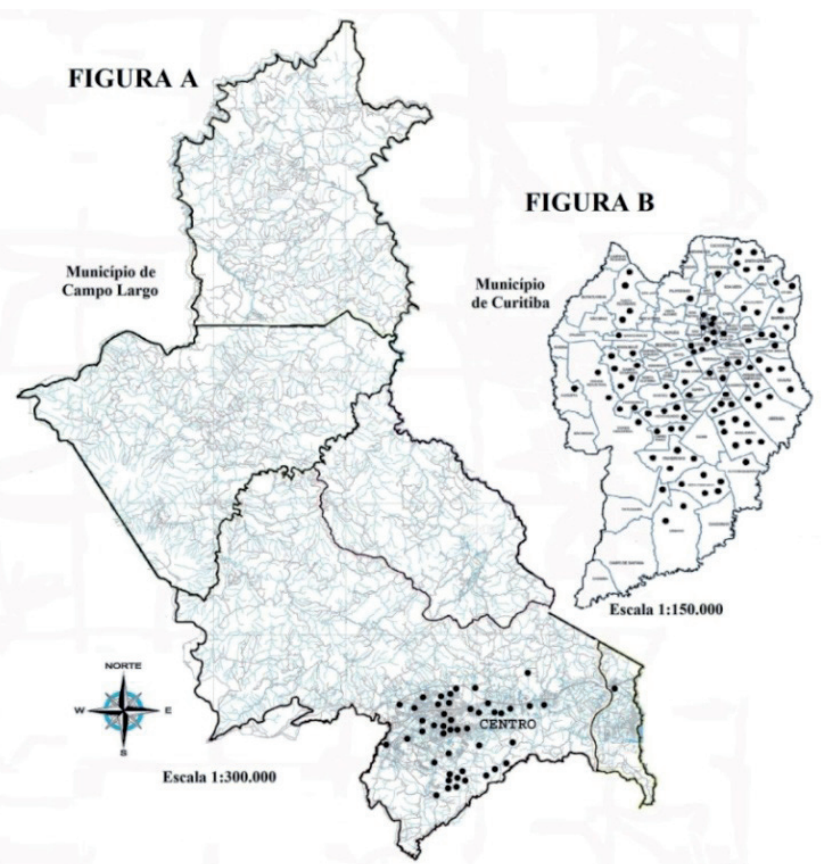

Figura1 - Localização geográfica de denúncias de maus-tratos contra animais no município de Campo Largo, registradas na Sociedade Protetora dos Animais de Campo Largo (Figura A), no período de 2001 até 2009, e no município de Curitiba (Figura B), registradas na Sociedade Protetora dos Animais de Curitiba, no período de 2003 a 2011, e na Rede de Defesa e Proteção Animal de Curitiba, no período de julho de 2009 a agosto de 2010 
é realizada feira de adoção de animais semanalmente, que caracteriza também uma oportunidade para informar a população sobre a possibilidade de denúncia de maus-tratos. O bairro Botiatuva apareceu como principal local de abandono de animais, provavelmente devido à presença da chácara da SPACL.

A distribuição geográfica dos casos de maus-tratos em Curitiba também demonstrou menor prevalência nas regiões mais afastadas do centro. A tendência ao maior número de suspeitas de maus-tratos na região central pode estar relacionada à maior ocorrência de maus-tratos ou ao fato da população local recorrer mais à denúncia. Ainda, o desconhecimento dos locais destinados ao recebimento de denúncias ${ }^{1}$, o desconhecimento da lei, a indiferença, o medo ou o descrédito na justiça são fatores que influenciam negativamente no processo de denúncia, o que eleva o índice de impunidade nos casos de violência contra animais e dificulta um conhecimento da distribuição real de casos de maus-tratos ${ }^{10}$. A evidência de que regiões da periferia das duas cidades estudadas apresentaram menor número de denúncias sugere a necessidade de implantação de campanhas educativas nas regiões periféricas dos municípios, com o objetivo de promover a guarda responsável. É importante fomentar ações de proteção animal e de orientação quanto aos cuidados a serem dispensados aos animais, inclusive informando a população sobre as possibilidades de realização de denúncias e aplicação das sanções penais aos infratores, as quais podem variar de multa a detenção ${ }^{3}$.
$\mathrm{Na}$ SPACL, as denúncias estudadas foram registradas em delegacias na forma de BO. A SPACL foi a responsável pela realização do $\mathrm{BO}$ em $67,2 \%$ dos casos (43/64), devido ao receio dos envolvidos. $\mathrm{Na}$ SPAC, 73,6\% (53/72) das denúncias foram encaminhadas para a Delegacia de Proteção ao Meio Ambiente, $16,7 \%$ (12/72) para delegacias regionais, 4,2\% (3/72) para o Ministério Público do Meio Ambiente, $2,8 \%(2 / 72)$ para o IAP e $2,8 \%$ (2/72) não foram informadas. A SPAC registrou BO para 73,6\% (53/72) das denúncias, 23,6\% (17/72) foram registradas por moradores e 2,8\% (2/72) anonimamente. Na RDPAC todas as denúncias foram realizadas pela população, sem o conhecimento pela RDPAC de encaminhamentos posteriores para delegacias.

$\mathrm{Na}$ SPACL, todas as denúncias foram contra pessoa física. Na SPAC, 87,9\% (51/58) das denúncias com informações sobre o denunciado (58/72) foram contra pessoa física e 12,1\% (7/58) incriminaram empresas, tais como criação e locação de cães, ou instituição de ensino. Na RDPAC, 78,0\% (46/59) das denúncias com informações sobre o denunciado (59/104) culparam pessoa física, 18,6\% (11/59) empresas de locação de cães, $1,7 \%$ (1/59) estabelecimento de comércio de animais e $1,7 \%$ (1/59) um estabelecimento de banho e tosa. A análise dos denunciados revelou que $72,5 \%$ foram homens (Tabela 1). Em estudo nos Estados Uni$\operatorname{dos}^{11}$ foi observada uma proporção de $95 \%$ dos acusados do sexo masculino, sendo consenso que pessoas do sexo masculino são mais propensas a cometer atos de maus-tratos contra animais ${ }^{8,12}$. Com relação aos

Tabela 1 - Gênero de denunciantes e denunciados em processos de maus-tratos contra animais envolvendo pessoa física, de acordo com os registros da Sociedade Protetora dos Animais de Campo Largo (SPACL), no período de 2001 até 2009, da Sociedade Protetora dos Animais de Curitiba (SPAC), de maio de 2003 a maio de 2011 e da Rede de Defesa e Proteção Animal de Curitiba (RDPAC), de julho de 2009 a agosto de 2010

\begin{tabular}{ccccc}
\hline & \multicolumn{2}{c}{$\begin{array}{c}\text { Porcentagem de denunciantes } \\
\text { (número absoluto) }\end{array}$} & \multicolumn{2}{c}{$\begin{array}{c}\text { Porcentagem de denunciados } \\
\text { (número absoluto) }\end{array}$} \\
\cline { 2 - 4 } & Sexo feminino & Sexo masculino & Sexo feminino & Sexo masculino \\
\hline SPACL & $76,2 \%(16)$ & $23,8 \%(05)$ & $27,1 \%(19)$ & $72,9 \%(51)$ \\
SPAC & $76,5 \%(13)$ & $17,6 \%(03)$ & $25,0 \%(14)$ & $75,0 \%(43)$ \\
RDPAC & $85,7 \%(36)$ & $11,9 \%(05)$ & $30,4 \%(14)$ & $69,6 \%(32)$ \\
\hline
\end{tabular}


denunciantes, $79,5 \%$ foram mulheres, também coerente com a literatura ${ }^{1}$.

Em 10\% (24/240) das denúncias foi possível observar informações sobre infratores violentos, que além de agredirem animais, agiam com violência contra seres humanos. Tais atos podem estar relacionados à exposição a ambientes com altas taxas de criminalidade, bem como em adultos que sofreram abuso sexual ${ }^{13}$ ou que foram punidos fisicamente durante a infância ${ }^{14}$. Nestes casos, há maior propensão para atos de maus-tratos contra animais, os quais por sua vez podem constituir um indicador de violência entre seres humanos ${ }^{15,16,17,18}$. Segundo Arkow ${ }^{19}$, quando animais são maltratados, pessoas estão em risco, e quando pessoas são maltratadas, animais estão em risco. Esta associação reforça a necessidade de uma cooperação interinstitucional ${ }^{1}$, segundo a qual os serviços sociais de atenção às famílias possam trabalhar em conjunto com os serviços de proteção animal, originando uma comunicação transversal na investigação de casos de maus-tratos.

Com relação às espécies animais, 82,9\% (203/240) das denúncias envolveram cães, seguido de cavalos $(8,6 \%$
- 21/240) e gatos (6,5\% - 16/240) (Tabela 2), similar a resultados dos Estados Unidos ${ }^{20}$. O maior número de relatos envolvendo cães pode ser explicado pela maior proximidade do ser humano com estes animais, por meio do vínculo como animais de companhia. Cerca de 59\% dos domicílios brasileiros possuem algum animal de estimação, sendo que em $44 \%$ deles há pelo menos um cão e em $16 \%$ pelo menos um gato ${ }^{21}$. O resultado também pode estar relacionado ao fato de que há uma maior quantidade de cães nas cidades estudadas que de outros animais. Segundo dados do IBGE ${ }^{22}$, em Campo Largo vivem 13206 bovinos e 2288 equinos, enquanto que se for considerada uma relação ser humano: cão de 4,0:123, o município conta com aproximadamente 30 mil cães. Em Curitiba, a proporção de cães em relação a bovinos e equinos é ainda maior, existindo 1092 bovinos e 432 equinos no município ${ }^{22}$ e cerca de 440 mil cães ${ }^{23}$. Da mesma forma, o menor número de denúncias envolvendo gatos (16/240) pode ser explicado pelo fato de cães serem os animais de companhia mais populares, apesar do aumento do número de gatos como animais de estimação nos últimos anos ${ }^{24}$. Apesar de um

Tabela 2 - Número de denúncias de maus-tratos por grupo animal e número de animais envolvidos em denúncias registradas na Sociedade Protetora dos Animais de Campo Largo (SPACL), no período de 2001 até 2009, da Sociedade Protetora dos Animais de Curitiba (SPAC), de maio de 2003 a maio de 2011 e da Rede de Defesa e Proteção Animal de Curitiba (RDPAC), de julho de 2009 a agosto de 2010

\begin{tabular}{|c|c|c|c|c|c|c|c|c|}
\hline \multirow{2}{*}{$\begin{array}{l}\text { Grupo } \\
\text { animal }\end{array}$} & \multicolumn{2}{|c|}{ SPACL } & \multicolumn{2}{|c|}{ SPAC } & \multicolumn{2}{|c|}{ RDPAC } & \multicolumn{2}{|c|}{ Total } \\
\hline & $\begin{array}{c}\mathrm{N}^{\circ} \mathrm{de} \\
\text { denúncias }\end{array}$ & $\begin{array}{l}\mathrm{N}^{\circ} \text { de } \\
\text { animais }\end{array}$ & $\begin{array}{c}\mathrm{N}^{\circ} \mathrm{de} \\
\text { denúncias }\end{array}$ & $\begin{array}{l}\mathrm{N}^{\circ} \text { de } \\
\text { animais }\end{array}$ & $\begin{array}{c}\mathrm{N}^{\circ} \mathrm{de} \\
\text { denúncias }\end{array}$ & $\begin{array}{l}\mathrm{N}^{\circ} \text { de } \\
\text { animais }\end{array}$ & $\begin{array}{c}\mathrm{N}^{\circ} \mathrm{de} \\
\text { denúncias }\end{array}$ & $\begin{array}{l}\mathrm{N}^{\circ} \text { de } \\
\text { animais }\end{array}$ \\
\hline Cão & $52(75,4 \%)$ & $99(76,2 \%)$ & $57(79,0 \%)$ & $123(83,1 \%)$ & $94(90,4 \%)$ & $207(87,7 \%)$ & $203(82,9 \%)$ & $429(83,5 \%)$ \\
\hline Gato & $09(13,0 \%)$ & $15(11,5 \%)$ & $02(3,0 \%)$ & $06(4,1 \%)$ & $05(4,8 \%)$ & $22(9,3 \%)$ & $16(6,5 \%)$ & $43(8,4 \%)$ \\
\hline Cavalo & $06(8,7 \%)$ & $06(4,6 \%)$ & $11(15 \%)$ & $14(9,5 \%)$ & $04(3,8 \%)$ & $03(1,3 \%)$ & $21(8,6 \%)$ & $23(4,5 \%)$ \\
\hline Boi & - & - & $01(1,5 \%)$ & $01(0,7 \%)$ & - & - & $01(0,4 \%)$ & $01(0,1 \%)$ \\
\hline Pombo & $01(1,4 \%)$ & $10(7,7 \%)$ & - & - & - & - & $01(0,4 \%)$ & $10(1,9 \%)$ \\
\hline Paca & $01(1,4 \%)$ & NA & - & & - & & $01(0,4 \%)$ & NA \\
\hline $\begin{array}{l}\text { Ganso e } \\
\text { calopsita }\end{array}$ & - & - & - & - & $01(1,0 \%)$ & $04(1,7 \%)$ & $01(0,4 \%)$ & $04(0,8 \%)$ \\
\hline $\begin{array}{l}\text { Ave e } \\
\text { coelho }\end{array}$ & - & - & $01(1,5 \%)$ & $04(2,7 \%)$ & - & - & $01(0,4 \%)$ & $04(0,8 \%)$ \\
\hline Abelha & - & - & - & - & $01(1,0 \%)$ & NA & $01(0,4 \%)$ & NA \\
\hline Total & $\begin{array}{c}69 \\
(100 \%)^{*}\end{array}$ & $\begin{array}{c}130 \\
(25,3 \%)^{\mathrm{a}}\end{array}$ & $\begin{array}{c}72 \\
(100 \%)\end{array}$ & $\begin{array}{c}236 \\
(45,9 \%)^{\mathrm{b}}\end{array}$ & $\begin{array}{c}104 \\
(100 \%)\end{array}$ & $\begin{array}{c}148 \\
(28,8 \%)^{c}\end{array}$ & $\begin{array}{c}245 \\
(100 \%)\end{array}$ & $\begin{array}{c}514 \\
(100 \%)\end{array}$ \\
\hline
\end{tabular}

* Total de 64 denúncias acrescido de cinco denúncias que envolveram mais de uma espécie animal

NA: Não se Aplica - relato de "vários" animais envolvidos. a , becEste total é parcial para o número de animais, pela inexistência de informaçoes exatas, mas sim o relato de "vários" animais envolvidos no caso de 31 denúncias. Dessa forma, os valores representam o número mínimo de indivíduos envolvidos 
acesso mais facilitado dos gatos às ruas,devido ao seu comportamento principalmente noturno e arbóreo eles podem ser menos vistos pela população. Também, o gato além de predador apresenta comportamento de presa, evitando demonstrar sinais de dor e doenças e procurando locais para se esconder quando ferido $^{25}$. Isso pode dificultar a visualização de maus-tratos contra esses animais. Assim, menor número de denúncias de maus-tratos contra gatos pode não refletir menor ocorrência de maus-tratos, sendo que Marlet e Maiorka ${ }^{26}$ relatam uma maior ocorrência de maus-tratos em gatos que em cães, proporcionalmente ao tamanho das populações dessas espécies.

Das 76 denúncias com informações sobre o tamanho dos cães, 64,5\%(49/76) envolveram cães de grande porte, o que pode estar relacionado ao alto número de denúncias envolvendo cães de guarda. Outros pesquisadore ${ }^{27}$ também encontraram maior ocorrência de maus-tratos em cães de grande porte se comparados a cães de pequeno porte, sugerindo que tal fato talvez se explique por esses cães serem mantidos normalmente na parte externa da casa, onde a população pode observá-los. No caso de cavalos, as denúncias podem estar relacionadas ao seu uso na coleta de lixo e materiais recicláveis em Curitiba. Por estarem sujei- tos a uma rotina de vida distinta daquela natural para a espécie, avaliações do seu uso podem revelar problemas relacionados ao seu grau de bem-estar e indicar a ocorrência de sofrimento ${ }^{28}$.

O número mínimo de indivíduos envolvidos em cada denúncia pode ser observado na tabela 2. Em 12,9\% (31/240) das denúncias não houve relato do número de animais envolvidos, mas sim o registro de grupos de indivíduos. A mediana de animais por denúncia foi 1,0, com mínima de um e máxima de 40 animais por denúncia. Tais resultados sugerem ser importante propiciar ao denunciante um formulário padrão no qual se insira um espaço fixo para relato do número de animais, informações sobre os animais envolvidos, como raça, idade e sexo, além da descrição da denúncia e endereço da ocorrência.

A classificação das causas de denúncias evidenciou que em 48,3\% (116/240) das denúncias houve mais de uma causa (Tabela 3). O número médio de causas por denúncia na SPACL, SPAC e RDPAC foi de 1,5 $\pm 0,7$, $3,6 \pm 1,0$ e $1,7 \pm 0,8$, respectivamente. Talvez o maior número de denúncias compreendendo somente uma ou duas causas decorra do fato que as pessoas relatam o que é mais notável quando observam um ato

Tabela 3 - Causas de denúncia de acordo com os registros na Sociedade Protetora dos Animais de Campo Largo (SPACL), no período de 2001 até 2009, da Sociedade Protetora dos Animais de Curitiba (SPAC), de maio de 2003 a maio de 2011 e da Rede de Defesa e Proteção Animal de Curitiba (RDPAC), de julho de 2009 a agosto de 2010

\begin{tabular}{|c|c|c|c|c|}
\hline \multirow{2}{*}{ Categorias de denúncias de maus-tratos } & \multicolumn{4}{|c|}{ Número de ocorrências por causa (porcentual) } \\
\hline & SPACL & SPAC & RDPAC & TOTAL \\
\hline Abandono & $21(21,2 \%)$ & $13(9,9 \%)$ & $32(18,1 \%)$ & $66(16,2 \%)$ \\
\hline Não fornecimento de alimento e água & $11(11,1 \%)$ & $18(13,7 \%)$ & $35(19,8 \%)$ & $64(15,7 \%)$ \\
\hline Morte do animal & $31(31,3 \%)$ & $19(14,5 \%)$ & $10(5,6 \%)$ & $60(14,7 \%)$ \\
\hline Ausência de atendimento veterinário & $5(5,1 \%)$ & $32(24,4 \%)$ & $18(10,2 \%)$ & $55(13,5 \%)$ \\
\hline Trauma físico, agressão & $13(13,1 \%)$ & $21(16 \%)$ & $13(7,3 \%)$ & $47(11,6 \%)$ \\
\hline Animal com restrição de espaço & $7(7,1 \%)$ & $9(6,9 \%)$ & $29(16,4 \%)$ & $45(11,1 \%)$ \\
\hline Outros fatores de inadequação ambiental & $4(4,0 \%)$ & $5(3,8 \%)$ & $18(10,2 \%)$ & $27(6,6 \%)$ \\
\hline Cães de guarda de empresas, negligência & - & $7(5,3 \%)$ & $10(5,6 \%)$ & $17(4,3 \%)$ \\
\hline Animal com acesso à rua sozinho & $5(5,1 \%)$ & - & $9(5,1 \%)$ & $14(3,4 \%)$ \\
\hline Abuso, uso excessivo & $1(1,0 \%)$ & $4(3,1 \%)$ & $2(1,1 \%)$ & $7(1,7 \%)$ \\
\hline Outros $^{\mathrm{a}}$ & $1(1,0 \%)$ & $3(2,3 \%)$ & $1(0,6 \%)$ & $5(1,2 \%)$ \\
\hline Total & $99(100 \%)$ & $131(100 \%)$ & $177(100 \%)$ & $407(100 \%)$ \\
\hline
\end{tabular}


de maus-tratos, deixando de relatar outros fatores que podem configurar causas adicionais de denúncia.

$\mathrm{O}$ abandono representou a causa mais comum de denúncia, com 16,2\% (66/407) das ocorrências, incluindo abandono em via pública e abandono na antiga residência por ocasião de mudança. Ao se aplicar a definição de maus-tratos ${ }^{29,30,31}$, o abandono pode ser considerado mau-trato, uma vez que submete o animal à falta de alimento e água, com prejuízos para sua saúde física e mental, associada a uma condição desconhecida e de variados riscos. $\mathrm{O}$ animal de estimação é dependente de seu responsável para atendimento de suas necessidades básicas. $\mathrm{O}$ abandono na antiga residência por ocasião de mudança, em alguns casos, resultou em morte por desnutrição e caquexia, exemplos sólidos de maus-tratos com grande magnitude de sofrimento. Considerando que os animais vertebrados são aceitos como seres sencientes ${ }^{32}$, pode-se sugerir que casos como estes envolvem sentimentos fortemente negativos.

O não fornecimento de alimento e água aos animais foi a segunda causa de denúncia, representando 15,7\% (64/407) das causas. Em muitos casos, o denunciante observou animais de guarda de empresas ou animais em residências sem alimentação adequada. Essas ocorrências são exemplos de negligência que, por definição, é a falha na provisão das necessidades básicas de um animal ${ }^{33}$. Outros exemplos incluem alimentação inadequada, falta de atendimento veterinário e o não fornecimento de abrigo e ambiente adequados, que foram também encontrados nesta pesquisa e que constituem formas muito comuns de maus-tratos ${ }^{33,34}$.

A morte foi a terceira causa de denúncia, representando a causa mais comum na SPACL $(31,3 \%)$, terceiro lugar na SPAC $(14,5 \%)$ e quinto lugar na RDPAC $(5,6 \%)$. A mais alta prevalência de morte nas denúncias da SPACL e da SPAC pode estar relacionada ao registro somente das denúncias consideradas de maior gravidade. Informações sobre a causa mortis não estavam disponíveis. Dos casos de morte, $27,9 \%$ foram relatados como intencionais por intoxicação exógena, seguidos de morte por desnutrição e caquexia $(13,1 \%)$ (Tabela 4). As mortes por intoxicação exógena intencional são relatadas com alta frequência também na literatura ${ }^{26,35}$.

A população pareceu sensibilizar-se frente a situações nas quais o animal se encontrava em ambiente inadequado, com restrição de espaço e abrigo, terceira e quarta causas de denúncias mais frequentes na

Tabela 4 -Causas de morte e sua frequência nas denúncias de maus-tratos registradas na Sociedade Protetora dos Animais de Campo Largo (SPACL), no período de 2001 até 2009, da Sociedade Protetora dos Animais de Curitiba (SPAC), de maio de 2003 a maio de 2011 e da Rede de Defesa e Proteção Animal de Curitiba (RDPAC), de julho de 2009 a agosto de 2010

\begin{tabular}{|c|c|c|c|c|}
\hline \multirow{2}{*}{ Causas de morte } & \multicolumn{4}{|c|}{ Número de ocorrências por causa de morte (porcentual) } \\
\hline & SPACL & SPAC & RDPAC & TOTAL \\
\hline Intoxicação exógena intencional & $12(38,7 \%)$ & $02(10,5 \%)$ & $03(27,2 \%)$ & $17(27,9 \%)$ \\
\hline Desnutrição e caquexia & $04(12,9 \%)$ & $04(21,1 \%)$ & - & $08(13,2 \%)$ \\
\hline Intencional, sem meio determinado & $04(12,9 \%)$ & $02(10,5 \%)$ & $02(18,2 \%)$ & $08(13,2 \%)$ \\
\hline Negligência de atendimento veterinário & $01(3,2 \%)$ & $04(21,1 \%)$ & $01(9,1 \%)$ & $06(9,8 \%)$ \\
\hline Espancamento & $01(3,2 \%)$ & $02(10,5 \%)$ & $01(9,1 \%)$ & $04(6,5 \%)$ \\
\hline Projétil & $03(9,7 \%)$ & - & - & $03(4,9 \%)$ \\
\hline Brigas com animais em via pública & $02(6,5 \%)$ & - & $01(9,1 \%)$ & $03(4,9 \%)$ \\
\hline Atropelamento intencional & $01(3,2 \%)$ & $01(5,3 \%)$ & $01(9,1 \%)$ & $03(4,9 \%)$ \\
\hline Esfaqueamento & $02(6,5 \%)$ & - & - & $02(3,3 \%)$ \\
\hline Enforcamento & - & $01(5,3 \%)$ & $01(9,1 \%)$ & $02(3,3 \%)$ \\
\hline Mutilação & - & $02(10,5 \%)$ & - & $02(3,3 \%)$ \\
\hline Afogamento & $01(3,2 \%)$ & - & - & $01(1,6 \%)$ \\
\hline Queimadura & - & $01(5,3 \%)$ & - & $01(1,6 \%)$ \\
\hline Óbito no serviço de banho e tosa & - & - & $01(9,1 \%)$ & $01(1,6 \%)$ \\
\hline TOTAL & $31(100 \%)$ & $19(100 \%)$ & $11(100 \%)$ & $61(100 \%)$ \\
\hline
\end{tabular}


RDPAC, respectivamente. Uma situação de confinamento de qualquer animal, que o impossibilite de exibir seu comportamento natural, constitui impedimento importante de seu bem-estar, restringindo diretamente a liberdade comportamental ${ }^{36}$. Talvez o grande número de residências e a alta densidade populacional de Curitiba provoquem limitação para a oferta de espaço adequado para os animais, diferente do que ocorre em Campo Largo. Duas denúncias registradas na RDPAC, que relataram cães submetidos a condições diárias de confinamento em canis pequenos, foram desconsideradas como maus-tratos. Alegou-se que os animais estavam bem alimentados e que o ambiente estava limpo e a denúncia não foi encaminhada para julgamento. Um diagnóstico técnico revelaria dados importantes sobre as Liberdades Comportamental, Ambiental e Psicológica relevantes para a resolução destes casos.

As agressões físicas intencionais representaram 11,6\% (47/407) das causas de denúncias (Tabela 3). Esta categoria incluiu casos de espancamento, facadas, abuso sexual, entre outros, situações nas quais o autor do crime submete o animal a uma variedade de ações que causam lesões corporais ${ }^{31}$. Na SPACL, a maioria destas ocorrências envolveu testemunha presencial e relato imediato para órgãos policiais oficiais. Espancamento, facadas e lesões por projétil também têm sido relatados como causas comuns de maus-tratos contra animais nos Estados Unidos ${ }^{20}$.

As denúncias apresentaram um perfil variado quanto à restrição das Cinco Liberdades, conforme o relato dos denunciantes (RD) e a avaliação técnica (AT) (Tabela 5). O número médio de liberdades afetadas por denúncia segundo o RD e a AT foi de 1,5 $\pm 1,0$ e 2,8 $\pm 1,1$, respectivamente. $\mathrm{O}$ estudo do $\mathrm{RD}$ revelou que em média $71,8 \%$ das ocorrências fizeram referência à liberdade sanitária, uma vez que os casos registrados envolveram com frequência animais feridos e doentes, seguido de apontamentos sobre a liberdade nutricional. Esse percentual foi compatível com a avaliação técnica $(72,3 \%)$, indicando que problemas físicos e de ordem sanitária são mais facilmente identificados pela população. Em contrapartida, a interpretação técnica sugeriu uma prevalência maior de comprometimento de todas as liberdades em relação ao relato do denunciante, especialmente sobre a liberdade psicológica, devido ao alto número de casos de abandono, falta de alimento, causas de morte e agressões; situações que denotam potencial sofrimento psicológico e distresse. Assim, os resultados sugerem que o enquadramento das situações nas quais se suspeita de maus-tratos pode ser aprimorado quando se insere o conhecimento técnico de profissional capacitado em bem-estar animal.

Tabela 5 - Comprometimento de cada liberdade baseado no relato do denunciante (RD) e na avaliação técnica (AT) das denúncias de maus-tratos contra animais registradasna Sociedade Protetora dos Animais de Campo Largo (SPACL), no período de 2001 até 2009, da Sociedade Protetora dos Animais de Curitiba (SPAC), de maio de 2003 a maio de 2011 e da Rede de Defesa e Proteção Animal de Curitiba (RDPAC), de julho de 2009 a agosto de 2010

\begin{tabular}{|c|c|c|c|c|c|c|c|}
\hline Local & & Total & $\mathrm{LN}$ & LA & LS & $\mathrm{LC}$ & LP \\
\hline \multirow[t]{2}{*}{ SPACL } & $\mathrm{RD}$ & 64 & $15(23,4 \%)$ & $14(21,9 \%)$ & $43(67,2 \%)$ & $11(17,2 \%)$ & $11(17,2 \%)$ \\
\hline & AT & 64 & $26(40,6 \%)$ & $26(40,6 \%)$ & $43(67,2 \%)$ & $15(23,4 \%)$ & $60(93,8 \%)$ \\
\hline \multirow[t]{2}{*}{ SPAC } & $\mathrm{RD}$ & 72 & $17(23,6 \%)$ & $07(9,7 \%)$ & $59(81,9 \%)$ & $05(6,9 \%)$ & $09(12,5 \%)$ \\
\hline & $\mathrm{AT}$ & 72 & $22(30,6 \%)$ & $17(23,6 \%)$ & $60(83,3 \%)$ & $22(30,6 \%)$ & $70(97,2 \%)$ \\
\hline \multirow{2}{*}{ RDPAC } & $\mathrm{RD}$ & 104 & $39(37,5 \%)$ & $30(28,8 \%)$ & $69(66,3 \%)$ & $27(26,0 \%)$ & $34(32,7 \%)$ \\
\hline & AT & 104 & $56(53,8 \%)$ & $53(51,0 \%)$ & $69(66,3 \%)$ & $42(40,4 \%)$ & $93(89,4 \%)$ \\
\hline \multirow{2}{*}{$\%^{\mathrm{a}}$} & $\mathrm{RD}$ & & $28,2 \%$ & $20,1 \%$ & $71,8 \%$ & $16,7 \%$ & $20,8 \%$ \\
\hline & $\mathrm{AT}$ & & $36,2 \%$ & $38,4 \%$ & $72,3 \%$ & $31,5 \%$ & $93,5 \%$ \\
\hline
\end{tabular}

LN: Liberdade Nutricional; LA: Liberdade Ambiental; LS: Liberdade Sanitária; LC: Liberdade Comportamental; LP:

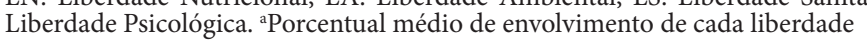


Todas as denúncias registradas na SPACL foram encaminhadas para delegacias e em 39,1\% (25/64), ocorreu inquérito policial seguido de ação penal com o parecer da Promotoria do Ministério Público. Foram deferidas pelo juiz e originaram sentença $80 \%$ (20/25) das referidas denúncias. Em todos os casos, o infrator teve a opção de escolher entre prestação de serviços voluntários à comunidade ou pagamento de multa, que variou de $\mathrm{R} \$ 100,00$ a $\mathrm{R} \$ 1.500,00$, em média $\mathrm{R} \$ 202,50 \pm 104,40$. Em estudo realizado nos Estados Unidos em uma entidade de proteção animal, foi observado que $44,4 \%$ de 268 casos de denúncia de maus-tratos físico contra animais resultaram em condenações e sentenças, que variaram de multa de 99 dólares a quatro meses de prisão ${ }^{20}$. O cenário jurídico estabelecido pelas denúncias na SPACL revelou maior porcentual de condenações em relação ao julgamento dos casos se comparado com o estudo norte-americano ${ }^{20}$. Porém, os casos documentados na SPAC sofreram pré-seleção quanto à gravidade, de forma que as denúncias estudadas correspondem às situações mais graves. Adicionalmente, se trata de um município com atuação marcante de uma entidade de proteção animal no atendimento de denúncias, o que pode sugerir uma situação privilegiada neste sentido. Entretanto, a realidade brasileira é mais complexa. A justiça atende, caso acionada, apenas $1 \%$ das situações de maus-tratos, considerando todos os sistemas de manutenção de animais ${ }^{8}$. Esta constatação reforça a necessidade de implantação de órgãos de polícia específicos para a proteção animal, como as Delegacias de Proteção Animal. Entre os 20\% (5/25) absolvidos estavam dois casos de abandono de animais em via pública. Os termos constantes da conclusão foram: "mesmo que houvesse sido juntada a prova de que o noticiado realmente abandonou filhotes de cão, não houve ocorrência de maus-tratos, já que maus-tratos se origina do verbo maltratar, que significa tratar com violência, bater em". Neste caso, a justiça desconsiderou que abandono é crime, contrariando o Decreto Federal $N^{\circ} 24.645 / 34$ e a própria definição de maus-tratos ${ }^{29,30,31}$ que configuram o abandono como maus-tratos. O parecer de um profissional capacitado em bem-estar animal em tais casos poderia ter subsidiado as posturas judiciais adotadas no sentido de aumentar a adequação da conclusão dos casos.

\section{Conclusão}

Foi possível conhecer o perfil das denúncias nas instituições estudadas, embora com limitações referentes à desuniformidade de conteúdo nas denúncias registradas. Os resultados indicaram maior participação de mulheres como denunciantes e homens como denunciados. Os cães foram a espécie mais citada e as principais causas de denúncia foram situações de abandono e negligência. Os resultados sugerem que a colaboração de profissional capacitado em bem-estar animal pode subsidiar o aprimoramento das deliberações em casos de suspeita de maus-tratos aos animais. A continuidade das pesquisas é necessária para uma maior compreensão do tema, especialmente envolvendo dados provenientes das delegacias e demais órgãos competentes.

\section{Agradecimentos}

À Aurora Guadagnin, presidente da Sociedade Protetora dos Animais de Campo Largo, a Soraya Simon, presidente da Sociedade Protetora dos Animais de Curitiba, e a Lucyenne Gisele Popp e Marcos Elias Traad da Silva, da Rede de Defesa e Proteção Animal de Curitiba, os sinceros agradecimentos pela indispensável colaboração na forma de cessão dos dados de denúncias. 


\section{Referências}

1.TAYLOR, N.; SIGNAL, T. D. Community Demographics and the Propensity to Report Animal Cruelty. Journal of Applied Animal Welfare Science, v. 9, n. 3, p. 201-210, 2006.

2. PEREIRA, R. $1^{\text {a }}$ Delegacia de Proteção Animal de São Paulo é instalada em Campinas. 2010. Disponível em: <http://www. itu.com.br/animais/noticia/1-delegacia-de-protecao-animalde-sao-paulo-e-instalada-em-campinas-20100323>. Acesso em: 26 ago. 2012.

3. BRASIL. Lei 9.605, de 12 de fevereiro de 1998. Diário Oficial da União, Brasília, DF, 13 fev. 1998. Seção 1, p. 29.

4. RODRIGUES, D. T. Observações sobre a proteção jurídica dos animais. Ciência Veterinária nos Trópicos, v. 13, p. 49-55, 2010. Suplemento, 1. Trabalho apresentado no II Congresso Brasileiro de Bioética e Bem Estar Animal, de 04 a 06 de agosto de 2010. Belo Horizonte, MG.

5. RDPAC. REDE DE DEFESA E PROTEÇÃO ANIMAL DA CIDADE DE CURITIBA. Resumo executivo do projeto rede de defesa e proteção animal da cidade de São Paulo. 2009. Disponível em: <http://www.protecaoanimal.curitiba.pr.gov. br/Publicacoes/Resumo-Plano-Municipal-Defesa-ProtecaoAnimal.pdf >. Acesso em: 12 abr. 2010.

6. FARM ANIMAL WELFARE COUNCIL (FAWC). Second report on priorities for research and development in farm animal welfare. London: Ministry of Agriculture, Fisheries and Food, 1993.

7. MOLENTO, C.F.M. Repensando as cinco liberdades. In: CONGRESSO INTERNACIONAL CONCEITOS EM BEMESTAR ANIMAL, 1., 2006, Rio de Janeiro.Anais... 2006. Disponível em: <http://www.labea.ufpr.br/publicacoes/pdf/W SPA\%202006\%20Cinco\%20Liberdades\%20portugu\%EAs\%20 -20REPENSANDO\%20AS\%20CINCO\%20LIBERDADES. pdf> Acesso em: 10 set. 2010.

8. AGNEW, R. The causes of animal abuse: a social psychological perspective. Theoretical Criminology, v. 2, p. 177-209, 1998.

9. HENSLEY, C.; TALLICHET, S. E. Animal cruelty motivations: assessing demographic and situational influences. Journal of Interpersonal Violence, v. 20, n. 11, p. 1429-1443, 2005.

10.LEVAI, L. F. Crueldade consentida: a violência humana contra os animais e o papel do ministério público no combate à tortura institucionalizada. 2002. Disponível em: <http://www. forumnacional.com.br/crueldade_consentida.pdf $>$. Acesso em: 04 abr. 2010.

11.VAUGHN, M. G.; FU, Q.; DELISI, M.; BEAVER, K. M.; PERRON, B. E.; TERRELL, K.; HOWARD, M. O. Correlates of cruelty to animals in the United States: Results from the National Epidemiologic Survey on Alcohol and Related Conditions. Journal of Psychiatric Research, v. 43, n. 15, p. 1213-1218, 2009.

12.HENSLEY, C.; TALLICHET, S. E. Effect of inmates' selfreported childhood and adolescent animal cruelty: motivations on the number of convictions for adult violent interpersonal crimes. International Journal of Offender Therapy and Comparative Criminology, v. 52, n. 2, p. 175-184, 2008.

13.CURRIE, C. L. Animal cruelty by children exposed to domestic violence. Child Abuse and Neglect, v. 30, n. 4, p. 425-435, 2006

14.MILLER, C. Childhood animal cruelty and interpersonal violence. Clinical Psychology Review, v. 21, n. 5, p. 735-749, 2001.

15. ASCIONE, F. R. Children who are cruel to animals: a review of research and implications for developmental psychopathology. Anthrozoos, v. 6, n. 4, p. 226-247, 1993.
16. ASCIONE, F. R. Animal abuse and youth violence. OJJDP. Juvenile Justice Bulletin, p. 1-15, 2001.

17.LOCKWOOD, R. Animal cruelty and human violence: the veterinarian's role in making the connection - The American experience. Canadian Veterinary Journal, v. 41, n. 11, p. 876$878,2000$.

18.MERZ-PEREZ, L.; HEIDE, K. M. Animal cruelty: pathway to violence against people. Lanham, MD: Rowman \& Littlefield, 2003.

19. ARKOW, P. The relationships between animal abuse and other forms of family violence. Family Violence \& Sexual Assault Bulletin, v. 12, n. 1/2, p. 29-34, 1996.

20.ARLUKE, A.; LUKE, C. Physical Cruelty Toward Animals in Massachusetts, 1975-1996. Society and Animals Journal, v. 5, n. 3, p. 195-204, 1997.

21.MORI, K. O verdadeiro mundo cão. 2004. Revista da Folha de São Paulo. Disponível em: <http://www.dogtimes.com.br/ revistafsp2.htm>. Acesso em: 20 set. 2011.

22.IBGE. Instituto Brasileiro de Geografia e Estatística. 2008. Disponível em: <www.ibge.gov.br>. Acesso em: 06 dez. 2010.

23.BIONDO, A. W.; KOBLITZ, E.; UTIME, R.; BONACIM, J. E.; FEITOSA, C.; VALEIXO, M.; CHI, K.D.; MOLENTO, C.F.M.Owned and semi-owned dog census in Curitiba and surroundings, Brazil. In: INTERNACIONAL SOCIETY OF APPLIED ETHOLOGY. NORTH AMERICAN REGIONAL MEETING, 37., 2006. Disponível em: <http://www.appliedethology.org/isaemeetings_files>. Acesso em: 16 nov. 2010.

24.GARCIA, R. C. M. Estudo da dinâmica populacional canina e felina e avaliação de ações para o equilíbrio dessas populações em áreas da cidade de São Paulo, SP, Brasil. 2009. $265 \mathrm{f}$. Tese (Doutorado em Medicina Veterinária) - Faculdade de Medicina Veterinária e Zootecnia, Universidade de São Paulo, São Paulo, 2009.

25.BEAVER, B.V. Feline behavior: a guide for veterinarians. Philadelphia: Saunders, 1992. 276 p.

26. MARLET, E. F.; MAIORKA, P. C. Análise retrospectiva de casos de maus-tratos contra cães e gatos na cidade de São Paulo. Brazilian Journal of Veterinary Research and Animal Science, v. 47, n. 5, p. 385-394, 2010.

27.DONLEY, L.; PATRONEK, G. J.; LUKE, C. Animal abuse in Massachusetts: a summary of case reports at the MSPCA and attitudes of Massachusetts veterinarians. Journal of Applied Animal Welfare Science, v. 2, n. 1, p. 59-73, 1999.

28.BURN, C. C.; DENNISON, T. L.; WHAY, H. R. Relationships between behaviour and health in working horses, donkeys, and mules in developing countries. Applied Animal Behaviour Science, v. 126, n. 3/4, p. 109-118, 2010.

29. FERREIRA, A. B. H. Novo dicionário da língua portuguesa. 2. ed. Rio de Janeiro: Nova Fronteira, 1995.

30.LEVAI, L. F. Direito dos animais. 2. ed. Campos do Jordão: Mantiqueira, 2004. 159 p.

31.MUNRO, R.; MUNRO, H. M. C. Animal abuse and unlawful killing: forensic veterinary pathology. China: Saunders, 2008. $106 \mathrm{p}$.

32.MOLENTO, C. F. M. Senciência Animal. Curitiba: Universidade Federal do Paraná,2005. Disponível em: <www. crmv-pr.org.br>. Acesso em: 10 set. 2010.

33. MERCK, M. D. Veterinary forensics: animal cruelty investigations. Iowa: Melinda Merck, 2007. p. 201-223, 327 p. 
34. CROOK, A. The CVMA Animal Abuse Position - How we got here. Canadian Veterinary Journal, v. 41, n. 8, p. 631-635, 2000.

35. MOLENTO, C. F. M.; LAGO, E.; BOND, G. B. Controle populacional de cães e gatos em dez Vilas Rurais do Paraná: resultados em médio prazo. Archives of Veterinary Science, v. 12, n. 3, p. 43-50, 2007.

36.WEBSTER, J. Animal welfare:limping towards Eden. Oxford: Blackwell Publishing, 2005. 283 p. 